\title{
Contrast effects in simultaneous discrimination learning
}

\author{
BEN A. WILLIAMS \\ University of California, San Diego, La Jolla, California 92093
}

\begin{abstract}
Pigeons were trained on a form discrimination that alternated across trials with an already-learned color discrimination, which was associated with different probabilities of reinforcement for different groups. The rate of acquisition of the form discrimination varied inversely with the rate of reinforcement for the color discrimination, thus demonstrating a "contrast effect" in simultaneous discrimination learning.
\end{abstract}

Contrast effects have occupied a major segment of the experimental literature in the area of learning over the past 20 years (cf. Mackintosh, 1974). In spite of its apparent ubiquity, however, few studies have examined contrast in procedures using dependent measures other than response rate or latency. The exceptions are studies that have varied magnitude of reinforcement in simultaneous discrimination procedures. Lawson (1957) and Schrier (1958) presented different magnitudes of reinforcement for different discriminations, either by presenting different magnitudes to different groups of subjects or by presenting the different magnitudes to the same subjects for different discriminations presented successively. Only when different magnitudes were presented to the same subject did the rate of learning vary as a function of magnitude. The apparent locus of the effect was that rate of learning with the smaller magnitudes was decreased when larger magnitudes were available for comparison. More recently, Eisenberger, Frank, and Park (1975) shifted rats in a straight alley from a small to a large magnitude of reinforcement, or from a large to a small magnitude, and then determined their preference for the alley associated with the shifts relative to a new alley associated with the same magnitude as used after the shift in reinforcement. In all cases, preference was predicted by a contrast effect. That is, subjects shifted from a high to low magnitude preferred the new alley, while subjects shifted from low to high preferred the alley associated with the shift.

There have been no studies of contrast in discrimination procedures where rate of reinforcement has been varied. That rate of reinforcement should produce effects similar to magnitude of

This research was supported by NSF Research Grant GB-42887. Portions of this research were reported at the meeting of the Psychonomic Society, November 1975. Requests for reprints should be addressed to the author, Psychology Department C-009, University of California at San Diego, La Jolla, California 92093. reinforcement is suggested by the similarity of their effects in other procedures (cf. Baum \& Rachlin, 1969; Catania, 1963). The present study examined contrast with choice as the dependent variable in a situation varying the rate of reinforcement. Pigeons first were trained on a color discrimination with a 1.0 or .33 probability of reinforcement for correct choices. A new form discrimination was then interspersed on some of the trials where both groups received $100 \%$ reinforcement for correct choices. The question was whether rate of learning the form discrimination varied as a function of rate of reinforcement associated with the already-learned color discrimination.

\section{METHOD}

\section{Subjects}

Twenty White Carneaux pigeons were maintained at $80 \%$ of their free-feeding body weights by additional feeding, when necessary, at the end of the daily experimental sessions. The subjects previously had been trained on a discrete-trial delayof-reinforcement procedure in a separate apparatus with red and green as stimuli (see Williams, 1975, Experiment 2, for details of procedure). The subjects in the different experimental conditions were matched with respect to the slight differences in their experimental histories.

\begin{abstract}
Apparatus
The apparatus was assembled from modular components produced by Coulborn Instruments, Inc. The interior chamber was $25 \mathrm{~cm}$ wide, $28 \mathrm{~cm}$ long, and $20 \mathrm{~cm}$ high, and was enclosed in a larger sound-attenuating chamber. On the aluminum front panel of the interior chamber were mounted two pigeon keys, $2.5 \mathrm{~cm}$ in diameter, that required a force of approximately $0.15 \mathrm{~N}$ for operation. Directly between and $18 \mathrm{~cm}$ below the keys was a $5 \times 5 \mathrm{~cm}$ aperture through which the birds were fed when the grain magazine was activated. A $28-\mathrm{V}$ houselight was also located between the two keys, at the top of the front panel. The stimuli for the keys were projected by standard 28-V IEE inline projectors. The color stimuli were diffuse lights occupying the entire surface of the keys. The form stimuli were a small white triangle and a small white circle, both approximately $.6 \mathrm{~cm}$ in diameter, projected onto the center of the otherwise darkened keys.
\end{abstract}

\section{Procedure}

For the first four sessions, all subjects were presented only the color discrimination. The positions of the two colors on the 


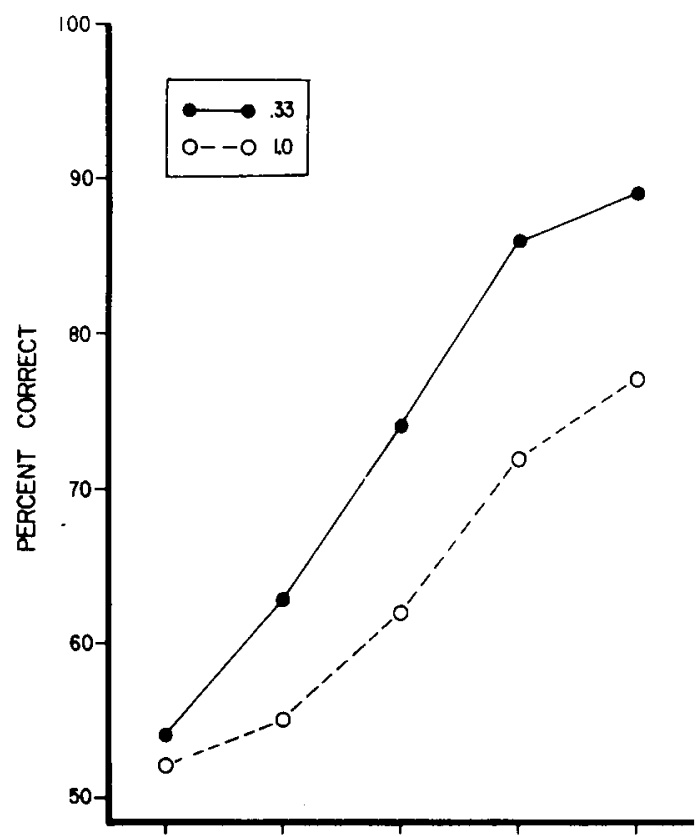

BLOCKS OF 3 SESSIONS

Figure 1. Percentage correct of responses to the form discrimination.

response keys were alternated quasi-randomly across trials. A response to the yellow color $(S+)$ resulted in 3-sec access to the mixed-grain reinforcer, followed by a 3 -sec intertrial interval (ITI) in which the keylights remained off and pecks had no effect. A response to the green light $(S-)$ resulted in a 6-sec ITI in which the keylights were turned off. Sixty trials were presented each session.

All subjects were treated the same on the first two of the four sessions and received reinforcement for each correct response. The subjects were then divided into two groups. The 1.0 group continued on the 1.0 probability of reinforcement schedule, while the .33 group now received reinforcement for only one-third of its correct color responses, as determined by a probability generator. Nonrewarded correct trials were treated exactly as incorrect trials, producing only the start of the 6-sec ITI.

On the fifth session the form stimuli were interspersed. For one-half of the subjects in each probability condition, the triangle was $\mathrm{S}+$ and the circle was $\mathrm{S}-$; for the remainder, the opposite contingencies were in effect. For all subjects a response to the correct form produced reinforcement on every trial while a response to the incorrect form produced only the 6-sec ITI. The reinforcement contingencies for the color trials remained unchanged. The form stimuli were interspersed on one-third of the trials, quasi-randomly as determined by a stepper, with the restriction that no two form trials occurred in succession. Twenty form trials and $\mathbf{4 0}$ color trials were presented each session. Training continued for 15 sessions.

\section{RESULTS}

The acquisition of the form discrimination is shown in Figure 1. The .33 condition produced faster learning. The results were analyzed statistically by a three-way analysis of variance (Reinforcement Probability by Stimulus by Blocks). The stimulus variable was included because the discrimination was learned more quickly when the circle served as
$\mathrm{S}+$. The results showed the Reinforcement Probability effect to be significant, $F(1,16)=8.03$, $p<.01$, as were the effects of the Stimulus variable, $F(1,16)=14.72, p<.01$, and the Blocks variable, $F(4,64)=48.42, p<.01$. The interaction between Reinforcement Probability and Blocks approached significance, $F(4,64)=2.30, p>.05$.

Differences in form discrimination occurred in spite of the fact that both groups continued to respond accurately on the color discrimination trials. Figure 2 shows the color discrimination performance. As shown by the unconnected points, the 1.0 condition produced slightly higher accuracy before the form discrimination was interspersed. Accuracy for both groups then declined when the form cues were introduced, with the 1.0 subjects affected more strongly. The results were analyzed by a two-way analysis of variance (Reinforcement Probability by Blocks). The Reinforcement Probability effect was significant, $F(1,18)=6.91, p<.05$, as was the Blocks variable, $F(4,72)=9.60, p<.01$. The interaction did not approach significance, $\mathrm{F}<1.0$.

The most likely cause of the differences in color discrimination was the development of a strong position habit on form discrimination trials. Control by positional cues apparently then occured on occasional color trials as well. Such position habits were less likely with the .33 condition, so accuracy of the color discrimination was less affected.

The data for individual subjects are shown in Table 1. The 1.0 condition produced more variability on the form discrimination, as three 1.0 subjects with the triangle as $\mathrm{S}+$ failed to show any sign of learning. In all cases, these subjects had adopted a strong position habit on the form trials.

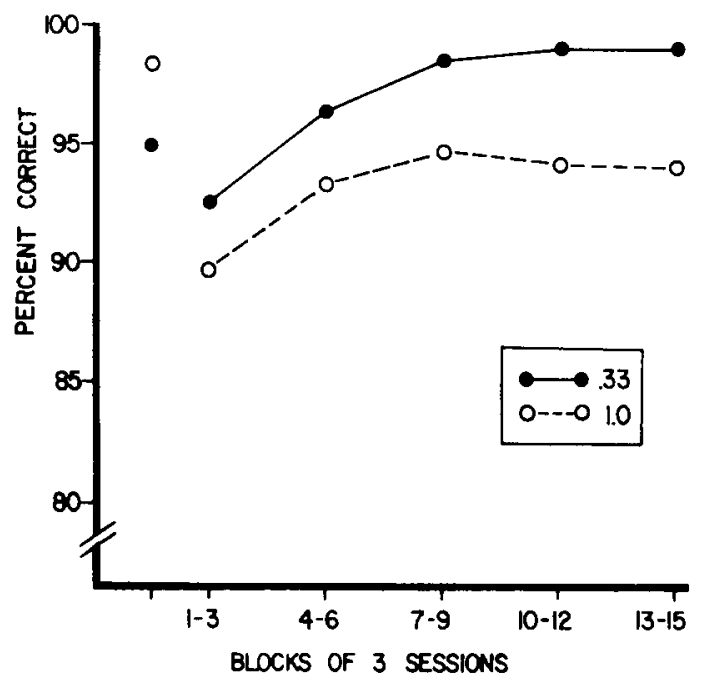

Figure 2. Percentage correct of responses to the color discrimination. The unconnected points represent the performance for the last day of training before the form discrimination was interspersed. 
Table 1

Percentage of Correct Trials for the Entire 15 Sessions of Training

\begin{tabular}{|c|c|c|c|c|c|}
\hline \multirow[b]{2}{*}{ Subject } & \multicolumn{2}{|c|}{ 1.0 Condition } & \multirow[b]{2}{*}{ Subject } & \multicolumn{2}{|c|}{33 Condition } \\
\hline & Form & Color & & Form & Color \\
\hline \multicolumn{6}{|c|}{ S+: Triangle } \\
\hline 1 & 72.7 & 91.6 & 1 & 69.0 & 94.8 \\
\hline 2 & 61.7 & 91.2 & 2 & 61.0 & 91.2 \\
\hline 3 & 51.0 & 92.0 & 3 & 63.0 & 99.4 \\
\hline 4 & 50.0 & 90.6 & 4 & 61.0 & 93.6 \\
\hline 5 & 50.0 & 85.8 & 5 & 73.0 & 97.4 \\
\hline \multicolumn{6}{|c|}{ S+: Circle } \\
\hline 1 & 77.3 & 97.2 & 1 & 76.3 & 98.0 \\
\hline 2 & 68.3 & 92.6 & 2 & 87.7 & 99.8 \\
\hline 3 & 80.3 & 96.8 & 3 & 81.0 & 98.6 \\
\hline 4 & 63.3 & 96.2 & 4 & 80.7 & 98.6 \\
\hline 5 & 57.3 & 97.4 & 5 & 76.3 & 99.2 \\
\hline
\end{tabular}

Note-Presented separately are accuracy for the form discrimination and accuracy for the color discrimination. The data are also divided according to whether the triangle or circle was the S+for the form discrimination.

Several 1.0 subjects performed at levels comparable to the .33 subjects. The overall difference between the .33 and 1.0 subjects was not dependent upon the complete failure of learning for some subjects, however, as there still was a significant difference between the conditions when the circle was the $\mathrm{S}+$, where all subjects in the 1.0 condition exhibited a significant degree of learning.

Also of interest from Table 1 is the relation between the form discrimination and color discrimination performance. Accuracy on the color trials was generally greater when the circle was the form $\mathrm{S}+$, for both groups, indicating that the easier the form discrimination, the less disruption of the color discrimination. Within a stimulus condition, however, there was no consistent relation across subjects between the color discrimination accuracy and the accuracy of the form discrimination.

\section{DISCUSSION}

The results demonstrated that the rate of acquisition of a simultaneous visual discrimination is inversely related to the rate of reinforcement of an interspersed, already-learned discrimination. The results are thus similar to previous effects found when magnitude of reinforcement has been varied for different discriminations presented to the same subject (cf. Schrier, 1958).

Before considering the possible explanations of the effect, it is important to exclude one alternative interpretation. One difference between the .33 and 1.0 conditions, quite apart from the probability of reinforcement associated with the color cues, was the average interreinforcement interval. It is possible that the longer interval with the .33 condition, as measured by the time between the preceding reinforcement and the onset of a form trial, was responsible for the facilitation. Such an explanation seems unlikely, however, since it implies that other situations with different interreinforcement intervals (i.e., when ITI is varied) should produce different degrees of learning. To the contrary, previous studies of the ITI in simultaneous discriminations typically have found no effect, even when pigeons have been used as subjects with form cues comparable to those of the present study (Biederman, 1967). In order to substantiate further that the interreinforcement interval, per se, was not responsible for the present effect, a second experiment was run which presented the triangle vs circle discrimination without the color discrimination interspersed. One group of six subjects received the 6-sec ITI used in Experiment 1; a second group of six subjects received an 18 -sec ITI (approximately the average interreinforcement interval for the .33 condition). Out of a possible 300 trials (five sessions of 60 trials each), the mean number of correct trials was 233 for the 6 -sec ITI and 220 for the 18-sec ITI. The difference was not statistically significant. Thus, the differences reported above did not depend upon the interreinforcement interval but, instead, were a function of the probability of reinforcement associated with the interspersed discrimination.

The major issue raised by the present resuits is the underlying basis of the obtained effect. Several possibly different explanations can be proposed. The simplest is that "context of reinforcement" affects the rate of learning just as it has been found to affect rate of responding in a variety of situations (cf. Herrnstein, 1970). One reservation about this interpretation is that it ignores a major feature of the data. The poor form discrimination in the 1.0 condition was correlated with the persistence of strong position habits. Thus, differences in form discrimination may not reflect differences in the rate of learning, per se, but rather differences in the relative degree of attention to form vs position. It is possible that the basis of the effect was that the position habit was acquired more quickly with the 1.0 condition, thus providing more potent interference in that condition.

A second experiment comes from the literature on observing behavior. D'Amato, Etkin, and Fazzaro (1968) have shown that the frequency of observing behavior increases when either extinction or reversal learning is instituted. The reduction in reinforcement for the color discrimination from 1.0 to .33 may have caused a similar increase in observing behavior for the .33 condition of the present study. This increase in observing behavior might then have extended to form trials and thus caused the .33 subjects to learn the form discrimination more quickly. The difficulty with this explana- 
tion is that it requires that the effects of reducing reinforcement for color discrimination extend over several sessions. Moreover, it ignores the possibility that a similar change in observing behavior could have occurred for the 1.0 subjects as well. That is, the introduction of the form discrimination resulted in a reduction in reinforcement on form trials to a $50 \%$ level (since they originally responded at chance). Since the subjects clearly discriminated whether form cues or color cues were present on a trial, this reduction in reinforcement on form trials should be expected to have generated observing behavior for the 1.0 subjects. Such observing behavior would not be expected for the .33 subjects since, for them, the form trials actually signaled a higher rate of reinforcement $(50 \%$ vs $33 \%)$. It appears, therefore, that the effects of observing behavior could explain the present data only if arbitrary assumptions were made about the determinants of the observing responses.

A third explanation is based on "blocking" (cf. Kamin, 1969). It is suggested by the finding that the poor form discrimination of 1.0 subjects was correlated with strong position habits on form trials. Although these position habits might be only the result of the poor form discrimination, it is also possible that they caused the poor form discrimination. Perhaps the position cues were associated with a greaier response strength under the 1.0 condition, and thus blocked control by the form cues more effectively. Greater association of position with reinforcement under the 1.0 condition is plausible because the same position cues were common to both the color and form discriminations. Thus, the average rate of reinforcement associated with the position stimuli (or any other stimuli common to both discriminations) was higher under the 1.0 condition. To the extent that this higher rate of reinforcement implies that the positional cues controlled a larger amount of response strength under the 1.0 condition, it follows from the blocking concept that acquisition of the form discrimination should be retarded. One difficulty with this interpretation is the requirement that substantial control by positional cues continue on color trials even after the color discrimination was highly learned.

Regardless of the eventual explanation of the above results, they do demonstrate that contrast effects are not limited to conditioning situations involving response rate or latency. To the extent that contrast represents a unitary phenomenon, therefore, any explanation must be generalizable to effects found with simultaneous discrimination learning.

\section{REFERENCES}

Baum. W. M., \& Rachlin. H. Choice as time allocation. Journal of the Experimental Analysis of Behavior. 1969, 12.861-874.

Biederman. G. Simultaneous discrimination: Parameters of reinforcement and ITI. Psychonomic Science, 1967, 8. 215-216.

Catania, A. C. Concurrent performances: A baseline for the study of reinforcement magnitude. Journal of the Experimental Analisis of Behavior. 1963, 6. 299-300.

D'Amato, M. R. Etkin, M. . Fazzaro. J. Cue-producing behavior in the capuchin monkey during reversal. extinction, acquisition. and overlearning. Journal of the Experimental Analysis of Behavior, 1968, 11. 425-433.

EISENBerger, R., Frank, M.. \& PARK, D. Incentive contrast of choice behavior. Joumal of Experimental Psychology: Animal Behavior Processes. 1975. 1. 346-354.

Herrnstein. R. J. On the law of effect. Journal of the Experimental A nalysis of Behavior, 1970, 13, 243-266.

KAMIN, L. J. Predictability, surprise, attention and conditioning. In R. Church \& B. Campbell (Eds.). Punishment and aversive behavior. New York: Appleton-Century-Crofts, 1969. Pp. 279-2\%.

LAWSON. R. Brightness discrimination performance and secondary reward strength as a function of primary reward amount. Journal of Comparative and Physiological Psychology. 1957. 50. 35-39.

Mackintosh. N. J. The psychology of animal learning. New York: Academic Press, 1974.

SChrier, A. M. Comparison of two methods of investigating the effects of amount of reward on performance. Journal of Comparative and Physiological Psychology. 1958, 51, 725-731.

Williams, B. A. The blocking of reinforcement control. Journal of the Experimental Analysis of Behavior, 1975. 24. 215-225.

(Received for publication July 27, 1976; revision accepted September 1, 1976.) 